\title{
An Effective Technique for Enhancing Direction Finding Performance of Virtual Arrays
}

\author{
Wenxing Li, Xiaojun Mao, Wenhua Yu, and Chongyi Yue \\ College of Information and Communication Engineering, Harbin Engineering University, Harbin 150001, China \\ Correspondence should be addressed to Xiaojun Mao; wwwmaoxiaojun@126.com
}

Received 8 July 2014; Revised 22 August 2014; Accepted 22 August 2014; Published 30 December 2014

Academic Editor: Dau-Chyrh Chang

Copyright ( 2014 Wenxing Li et al. This is an open access article distributed under the Creative Commons Attribution License, which permits unrestricted use, distribution, and reproduction in any medium, provided the original work is properly cited.

\begin{abstract}
The array interpolation technology that is used to establish a virtual array from a real antenna array is widely used in direction finding. The traditional interpolation transformation technology causes significant bias in the directional-of-arrival (DOA) estimation due to its transform errors. In this paper, we proposed a modified interpolation method that significantly reduces bias in the DOA estimation of a virtual antenna array and improves the resolution capability. Using the projection concept, this paper projects the transformation matrix into the real array data covariance matrix; the operation not only enhances the signal subspace but also improves the orthogonality between the signal and noise subspace. Numerical results demonstrate the effectiveness of the proposed method. The proposed method can achieve better DOA estimation accuracy of virtual arrays and has a high resolution performance compared to the traditional interpolation method.
\end{abstract}

\section{Introduction}

Array signal processing has a wide range of applications in radar, communications, sonar, and acoustics. Interpolation or mapping technique from a real antenna array to a virtual antenna array is a popular topic in array signal processing [1]. Virtual array interpolation (mapping) technique was introduced in 1980s [2-5]. Virtual array transformation can map an arbitrary planar structure antenna array to be a uniform linear array- (ULA-) type array and can increase the degrees of freedom (DOF) of an antenna array, which is widely used in the DOA estimation and adaptive beamforming $[6,7]$.

There exist some efficient DOA estimation algorithms such as "root-MUSIC" and "root-WSF", which are normally restricted to the ULA geometry. These algorithms cannot be used for the conformal arrays or uniform circular array (UCA) directly; however, the interpolation transform technique can solve this problem efficiently [8]. In Friedlander's virtual array transformation (VAT) method [9], an arbitrary shaped antenna array can be transformed into a desired virtual antenna array by interpolating the interested scanning sector. The basic idea of Friedlander's VAT is to divide the spatial region into several subregions, and then by taking interpolation in the array scanning sector of interest, the manifold of the virtual array can be obtained by the linear interpolation of the manifold of the real array. The transform matrix is computed as the least squares solution.

The performance of array interpolation in the DOA estimation has been reported in the literature [10-12]. Although the array interpolation approach has some attractive properties $[13,14]$, an essential shortcoming of the method is that it often introduces mapping errors that cause bias in the DOA estimation. Hence, the DOA estimations are not statistically optimal.

Pesavento et al. developed a robust interpolation method in [15]. The method ensures that the interpolation transform error of the selected sectors is minimal, while the conversion outside region sets up multiple "stop bands" to make the conversion error less than a criterion.

Hyberg et al. proposed a geometrical interpretation method of a Taylor series expansion of the DOA estimator criterion function to derive an alternative design of the mapping matrix $[16,17]$. The proposed design considers the orthogonality between the manifold mapping errors and certain gradients of the estimator criterion function. This bias-minimizing theory was extended to not only minimize bias but also consider finite sample effects due to noise reducing the DOA mean-square error (MSE). DOA MSE is 


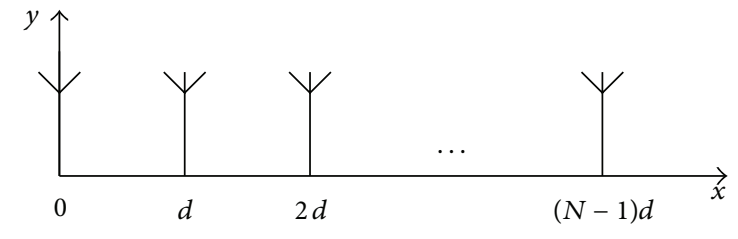

FIGURE 1: The structure of ULA.

not reduced by minimizing the size of the mapping errors, but instead by rotating the errors and the associated noise subspace into the optimal directions related to a certain gradient of the DOA estimator criterion function in [17]. The two methods show the superior performance on the DOA MSE reduction of array interpolation but its derivation computed procedure is complex.

This paper proposed a novel method to reduce the DOA bias of virtual interpolation. Using the projection concept, project the transformation matrix onto the real array covariance matrix to enhance the signal subspace, which improves the orthogonality between the signal and noise subspace. The proposed method is efficient and easy to realize. Numerical simulations verify that this method can get better estimation accuracy and has a high resolution performance compared to the traditional interpolation method.

\section{Signal Model}

Considering an omnidirectional array with $N$ elements illuminated by $M$ narrow band signals, the distance of the array elements is $d$, as shown in Figure 1.

The signal $s_{k}(t)$ is incident in the direction $\theta_{k}$; the received signal $\mathbf{X}$ can be expressed as follows:

$$
\mathbf{X}(t)=\mathbf{A S}(t)+\mathbf{N}(t)
$$

where $\mathbf{X}(t)=\left[x_{1}(t), x_{2}(t), \ldots, x_{N}(t)\right]^{T}$ is a $N \times 1$ snap data vector. $\mathbf{S}(t)=\left[s_{1}(t), s_{2}(t), \ldots, s_{M}(t)\right]^{T}$ is a vector containing the complex signal envelops of $M$ narrow-band signal sources. $\mathbf{N}(t)=\left[n_{1}(t), n_{2}(t), \ldots, n_{N}(t)\right]^{T}$ is a vector of zero-mean spatial white sensor noise of variance $\sigma_{n}^{2}$; $\mathbf{A}$ is an array manifold matrix; namely, $\mathbf{A}=\left[\mathbf{a}\left(\theta_{1}\right), \mathbf{a}\left(\theta_{2}\right), \ldots, \mathbf{a}\left(\theta_{M}\right)\right]$, where $\mathbf{a}\left(\theta_{1}\right)=\left[1, e^{j \beta_{k}}, \ldots, e^{j(N-1) \beta_{k}}\right]^{T}, k=1,2, \ldots, M$ represents a steering vector in the $\theta_{k}$ direction, and $\beta_{k}$ is the phase difference that can be represented as

$$
\beta_{k}=\frac{2 \pi}{\lambda} d \sin \left(\theta_{k}\right)
$$

Assume that the signal and noise are linearly independent, and then the data covariance is written in the format below:

$$
\mathbf{R}=E\left\{\mathbf{X}(t) \mathbf{X}^{H}(t)\right\}=\mathbf{A R}_{s} \mathbf{A}^{H}+\sigma_{n}^{2} \mathbf{I},
$$

where $E\{\}$ denotes the expectation operator; $\mathbf{R}_{s}=$ $E\left\{S(t) S^{H}(t)\right\}$ represents the autocorrelation matrix of signal complex envelops. $\sigma_{n}^{2}$ is the noise power; $\mathbf{I}$ is the unit matrix; and $(\cdot)^{H}$ denotes the matrix conjugate transposition.

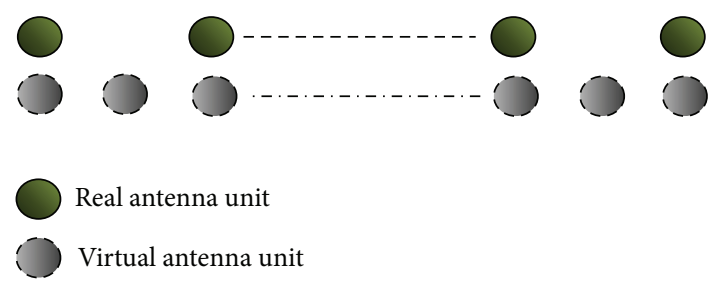

FIGURE 2: Real antenna and interpolated arrays.

In practice, the desired signal is often present in the snapshots. The sample array covariance matrix can be expressed as follows:

$$
\widehat{\mathbf{R}}=\frac{1}{K} \sum_{i=1}^{K} \mathbf{X}(i) \mathbf{X}^{H}(i)
$$

where $K$ is number of snapshots. VAT is based on interpolation technique [8] in which the entire antenna array scanning vector is divided into several subregions, and the subregion of interest will be segmented through a certain transformation to realize the mapping from the original array to the corresponding virtual array.

\section{Conventional Interpolated Array}

Consider a real ULA transformed into a virtual ULA via array interpolation, as illustrated in Figure 2.

Assume that there is a signal located in the region $\Theta$; we equally divide $\Theta$ into

$$
\Theta=\left[\theta_{l}, \theta_{l}+\Delta \theta, \theta_{l}+2 \Delta \theta, \ldots, \theta_{r}-\Delta \theta, \theta_{r}\right],
$$

where $\theta_{l}$ and $\theta_{r}$ are the left and right boundary of region $\Theta$, respectively. $\Delta \theta$ is size of the interpolation step, which is determined by the specified accuracy.

The real array manifold matrix in the chosen area can be expressed as follows:

$$
\begin{gathered}
\mathbf{A}=\left[\mathbf{a}\left(\theta_{l}\right), \mathbf{a}\left(\theta_{l}+\Delta \theta\right), \mathbf{a}\left(\theta_{l}+2 \Delta \theta\right), \ldots,\right. \\
\left.\mathbf{a}\left(\theta_{r}-\Delta \theta\right), \mathbf{a}\left(\theta_{r}\right)\right],
\end{gathered}
$$

where $\mathbf{a}\left(\theta_{l}\right)$ represents the steering vector of a real array in the $\theta_{l}$ direction. The array manifold matrix of virtual array in the same area $\Theta$ is expressed as follows:

$$
\begin{gathered}
\overline{\mathbf{A}}=\left[\overline{\mathbf{a}}\left(\theta_{l}\right), \overline{\mathbf{a}}\left(\theta_{l}+\Delta \theta\right), \overline{\mathbf{a}}\left(\theta_{l}+2 \Delta \theta\right), \ldots,\right. \\
\left.\overline{\mathbf{a}}\left(\theta_{r}-\Delta \theta\right), \overline{\mathbf{a}}\left(\theta_{r}\right)\right],
\end{gathered}
$$

where $\overline{\mathbf{a}}\left(\theta_{l}\right)$ represents the steering vector of a virtual array in the $\theta_{l}$ direction. There must exist a mapping relationship between the real and the virtual array vectors. Then an interpolation matrix $\mathbf{B}$ is designed to satisfy the least square; that is,

$$
\min _{\mathbf{B}}\left\|\mathbf{B}^{H} \mathbf{A}-\overline{\mathbf{A}}\right\|_{F}^{2}
$$


where $\|\cdot\|_{F}$ denotes the Frobenius norm for a matrix. The real and the virtual array manifold vectors satisfy the following relationship:

$$
\mathbf{B}^{H} \mathbf{A}(\theta)=\overline{\mathbf{A}}(\theta) \quad \theta \in \Theta .
$$

And their steering vectors satisfy the following equation:

$$
\mathbf{B}^{H} \mathbf{a}(\theta)=\overline{\mathbf{a}}(\theta) \quad \theta \in \Theta .
$$

When the number of a transformed array is greater than the actual number of antenna elements and the matrix $\overline{\mathbf{A}}$ has a nonzero condition value, by solving (8) the virtual transformation matrix $\mathbf{B}$ is

$$
\mathbf{B}=\left(\mathbf{A} \mathbf{A}^{H}\right)^{-1} \mathbf{A} \overline{\mathbf{A}}^{H} .
$$

Define the transformation error

$$
E(\mathbf{B})=\frac{\min _{\mathbf{B}}\left\|\mathbf{B}^{H} \mathbf{A}-\overline{\mathbf{A}}\right\|_{F}}{\|\overline{\mathbf{A}}\|_{F}} .
$$

In an ideal case, there is no error in the virtual transformation; $E(\mathbf{B})$ should be zero. However, in practice, since interpolation points in the transformation area infinities are limited, the interpolation operation often introduces mapping errors. These preprocessing techniques often introduce mapping bias and excess variance in the DOA estimations. Hence, the estimations are not statistically optimal [6].

\section{Modified Interpolated Method}

In this section, we describe a modified interpolation algorithm. We set the data covariance matrix of the real array $\widehat{\mathbf{R}}$ as a projection matrix. After obtaining the transform matrix $\mathbf{B}$, according to (11), we reconstruct the transformation matrix $\overline{\mathbf{B}}$ by projecting it to the sample array covariance matrix

$$
\overline{\mathbf{B}}=\widehat{\mathbf{R}} \mathbf{B} \text {. }
$$

For a given transformation matrix $\overline{\mathbf{B}}$, we can compute the covariance matrix of a virtual antenna array

$$
\begin{aligned}
\overline{\mathbf{R}} & =\overline{\mathbf{B}} \mathbf{A} \mathbf{R}_{s} \mathbf{A}^{H} \overline{\mathbf{B}}^{H}+\overline{\mathbf{B}}\left(\sigma_{n}^{2} \mathbf{I}\right) \overline{\mathbf{B}}^{H} \\
& =\widehat{\mathbf{R}} \mathbf{B} \mathbf{A} \mathbf{R}_{s} \mathbf{A}^{H} \mathbf{B}^{H} \widehat{\mathbf{R}}^{H}+\sigma_{n}^{2} \widehat{\mathbf{R}} \mathbf{B} \mathbf{B}^{H} \widehat{\mathbf{R}}^{H} .
\end{aligned}
$$

The above procedure can enhance the signal components in the virtual covariance matrix $\overline{\mathbf{R}}$ and improve the orthogonality between the signal and the noise subspace.

We can clearly see that $\overline{\mathbf{B B}}^{H} \neq \mathbf{I}$, which implies that the original white noise turns into the colored noise after the virtual transformation. For most DOA estimation algorithms can only work when the background noise is Gaussian white noise, and the colored noise must be prewhitened. Define the transformation matrix as

$$
\mathbf{T}=\left(\overline{\mathbf{B}}^{H} \overline{\mathbf{B}}\right)^{-1 / 2} \overline{\mathbf{B}}^{H}
$$

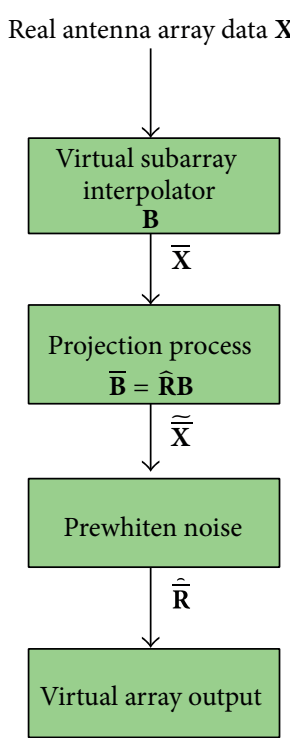

FIGURE 3: Construction of modified interpolated approach.

The real antenna array steering vector $\mathbf{a}(\theta)$ and the virtual array steering vector $\overline{\mathbf{a}}(\theta)$ have the following relationship:

$$
\operatorname{Ta}(\theta)=\left(\overline{\mathbf{B}}^{H} \overline{\mathbf{B}}\right)^{-1 / 2} \overline{\mathbf{a}}(\theta)=\overline{\overline{\mathbf{a}}}(\theta) \quad \theta \in \Theta .
$$

After the noise prewhitening above, the covariance matrix of the virtual antenna can be computed by using the transformation matrix $\mathbf{T}$.

Consider

$$
\overline{\overline{\mathbf{R}}}=\mathbf{T R} \mathbf{T}^{H}=\overline{\overline{\mathbf{A}}} \mathbf{R}_{s} \overline{\overline{\mathbf{A}}}^{H}+\sigma_{n}^{2} \mathbf{I} .
$$

Therefore, the covariance matrix of the virtual antenna is obtained, and the application of a direction finding estimator to (17) is straightforward [6].

To summarize the modified interpolation transformation technique, the transformation procedure is shown in Figure 3.

In this paper, the multiple signal classification (MUSIC) algorithm is used to estimate the DOA. MUSIC algorithm is a high resolution technique based on exploiting the eigenstructure of an input covariance matrix. We decompose the autocorrelation matrix into signal and noise subspaces. The covariance matrix $\overline{\bar{R}}$ can be written as

$$
\overline{\overline{\mathbf{R}}}=\mathbf{U}_{S} \Sigma_{S} \mathbf{U}_{S}^{H}+\mathbf{U}_{N} \Sigma_{N} \mathbf{U}_{N}^{H},
$$

where $\mathbf{U}_{S}$ represents the signal subspace; $\mathbf{U}_{N}$ represents the noise subspace; $\Sigma_{S}=\operatorname{diag}\left\{\lambda_{1}, \lambda_{2}, \ldots, \lambda_{M}\right\}$ is the signal eigenvalue; $\Sigma_{N}=\operatorname{diag}\left\{\lambda_{M+1}, \lambda_{M+2}, \ldots, \lambda_{N}\right\}$ is the noise eigenvalue. The noise subspace $\mathbf{U}_{N}$ is orthogonal to all $M$ signal steering vectors. The spectrum of the MUSIC algorithm is given by

$$
P_{\text {MUSIC }}=\frac{1}{\mathbf{a}^{H}(\theta) \mathbf{U}_{N} \mathbf{U}_{N}^{H} \mathbf{a}^{H}(\theta)}=\frac{1}{\left\|\mathbf{U}_{N}^{H} \mathbf{a}(\theta)\right\|} .
$$


TABLE 1: Comparison of the two methods.

\begin{tabular}{lccc}
\hline & Notion of DOA RMSE reduction & $\begin{array}{c}\text { Computational complexity } \\
\text { of mapping matrix T }\end{array}$ & $\begin{array}{c}\text { Additional prior } \\
\text { information compared to } \\
{[8]}\end{array}$ \\
\hline Array interpolation of [17] & $\begin{array}{c}\text { Rotate the mapping errors and noise } \\
\text { subspace into optimal directions relative } \\
\text { to a certain gradient of the DOA } \\
\text { estimator criterion function }\end{array}$ & $O\left(2(2 \bar{N}+1)^{2} N_{\text {cal }}^{2} \bar{N} N\right)$ & $\begin{array}{c}\text { Complex gradient of } \\
\text { criterion of the used } \\
\text { estimator }\end{array}$ \\
\hline Proposed method & $\begin{array}{c}\text { Project the transformation matrix on the } \\
\text { real array covariance matrix to strengthen } \\
\text { thesignal subspace }\end{array}$ & $O\left(\bar{N} N^{3}\right)$ & None \\
\hline
\end{tabular}

If $\theta$ is equal to DOA, the noise subspace $\mathbf{U}_{N}$ is orthogonal to the signal steering vectors and $\left\|\mathbf{U}_{N}^{H} \mathbf{a}(\theta)\right\|$ becomes zero when $\theta$ is a signal direction and the denominator is identical to zero. It is obvious that in practice $\mathbf{U}_{N}^{H} \mathbf{a}(\theta) \neq 0$ due to finite samples. If this happens the performance of MUSIC algorithm will not be optimal.

Now, we can summarize the modified VAT procedure as follows.

Step 1. Compute the real array covariance matrix $\widehat{\mathbf{R}}$.

Step 2. Compute the real array manifold $\mathbf{A}(\theta)$ and virtual array manifold $\overline{\mathbf{A}}(\theta)$ and then compute the transformation matrix B using (11).

Step 3. Take the projection operation to get the new transformation matrix $\overline{\mathbf{B}}$ using $\overline{\mathbf{B}}=\widehat{\mathbf{R}} \mathbf{B}$.

Step 4. Compute the covariance matrix $\overline{\overline{\mathbf{R}}}$ of the virtual array from the covariance matrix $\widehat{\mathbf{R}}$ of the real array.

Step 5. Apply the MUSIC algorithm to the covariance $\overline{\bar{R}}$ in (19).

We compare the proposed method with Hyberg's method in [17], which is selected for comparison because of its superior performance on DOA mean-square error (MSE) reduction of array interpolation. In [17], the authors proposed a design algorithm for the mapping matrix that minimized the DOA estimate bias. The MSE-minimizing mapping matrix $T$ is designed as

$$
\begin{aligned}
\mathbf{T}_{\mathrm{opt}}=\arg \min _{\mathbf{T}}\left\{\sum_{i=1}^{N_{\mathrm{cal}}}(1-\mu)\left\|\Delta e_{v}^{(i)}\right\|^{2}\right. \\
+\frac{\mu}{\ddot{V}^{2}\left(\theta^{(i)}, e_{v}^{(i)}\right)} \\
\times\left[4\left(\operatorname{Re}\left\{g_{v}^{(i)} \Delta e_{v}^{(i)}\right\}\right)^{2},\right. \\
\left.\left.+\sum_{k=2}^{m} 2 \alpha^{(i)}\left|g_{v}^{(i)} \mathbf{T}^{H} e_{k}^{(i)}\right|^{2}\right]\right\},
\end{aligned}
$$

where $\mu$ is a weighting factor $(0 \leq \mu<1)$ and $g_{v}^{(i)} \triangleq g_{v}\left(\theta^{(i)}\right)$ are the gradient vectors. $N_{\text {cal }}$ is the number of calibration directions. In general, the superscript (i) means that the corresponding quantity should be computed as if there were a single source in the $i$ th calibration direction (see [17] for details). DOA MSE is not reduced by minimizing the size of the mapping errors but instead by rotating these errors and the associated noise subspace into optimal directions relative to a certain gradient of the DOA estimator criterion function. We can clearly see that criterion in (20) is a quadratic function of the elements of $\mathbf{T}$. The characteristics of the method of [17] and the proposed method are listed in Table 1.

The comparisons of the two methods are given in Table 1 where we can see that method in [17] is much more complex than our method, which need to calculate the complex gradient of criterion of the used estimator at first compared to [8]. The calculation of references [17] is more than eight times higher than the proposed method. Simulation is conducted to evaluate the performance of the different methods.

\section{Numerical Examples}

In this section, the estimation accuracy of the proposed interpolation method and the conventional approach $[3,8,9]$ is evaluated through numerical simulations.

Numerical Experiment 1. The real array is uniform and linear with 4 elements and the element space is $\lambda$. The nondirectional noise is spatial white Gaussian with a unit variance. The virtual antenna array is uniform and linear array with 8 elements and element space $\lambda / 2$. There are four independent signals arriving from the directions $-55.0^{\circ}$, $-50.0^{\circ}, 10.0^{\circ}$, and $15.0^{\circ}$. The signal-to-noise ratio (SNR) of the six signals is $10 \mathrm{~dB}$ and the virtual transform sector is $\left[-60^{\circ},-40^{\circ}\right] \cup\left[0^{\circ}, 20^{\circ}\right]$. The step size is $0.1^{\circ}$. The number of snapshots is 128. 200 Monte Carlo runs are used to obtain each point.

The simulation results of conventional method and the proposed method are shown in Figure 4. It can be seen from the Figure 4 that the real array has 3 DOFs, which can process 3 signals at most, while the virtual antenna array has 8 elements with 7 DOFs and can process more than 3 signals. But the conventional interpolation method fails to distinguish the two close signals $\left(-55.0^{\circ},-50.0^{\circ}\right)$ and $\left(10.0^{\circ}, 15.0^{\circ}\right)$ for its large transform errors. The modified 


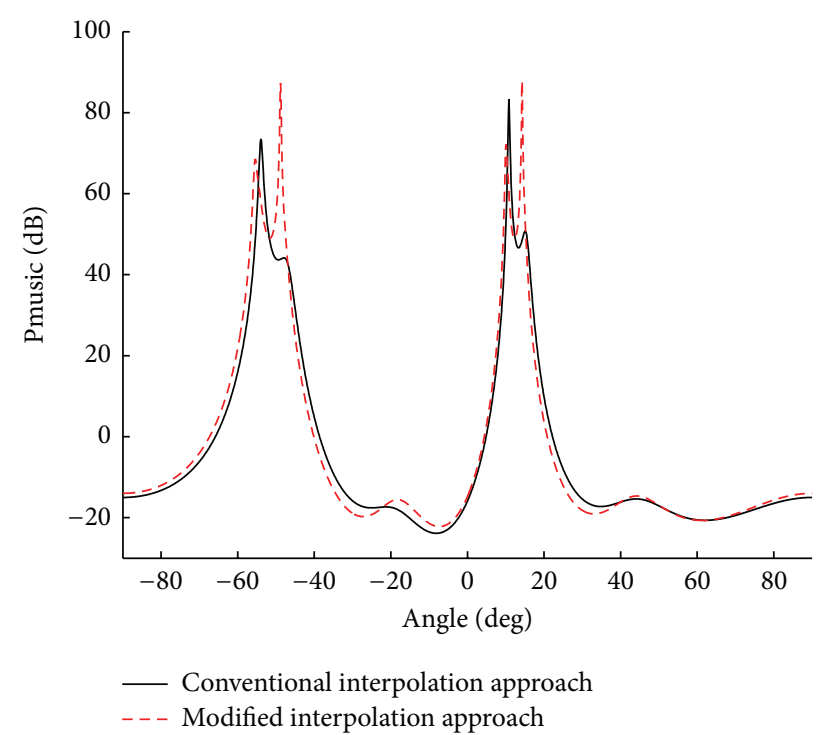

FIGURE 4: Spatial spectrum of MUSIC algorithm for two methods comparison.

interpolation method can distinguish the two very close signals. The DOA finding results is $\left(-55.4^{\circ},-49.2^{\circ}, 10.1^{\circ}\right.$, and $14.4^{\circ}$ ), and the result is accurate. We also can see that the modified interpolation method can still work in the case of the number of signals exceeding the DOFs of actual array and the resolution is improved compared to the conventional interpolation method.

Numerical Experiment 2. We consider a uniform and linear array with 4 elements and the element space $\lambda$. The nondirectional noise is spatial white Gaussian with a unit variance. The virtual antenna array is also uniform and linear array with 8 elements and element space $\lambda / 2$. Two independent signals arrive from the directions $0.0^{\circ}$ and $5.0^{\circ}$ and the virtual transformation area is $\left[-5^{\circ}, 10^{\circ}\right]$, and the step size is $0.1^{\circ}$. The SNR of the two signals is $10 \mathrm{~dB}$. All SNR values are referred as per antenna element, and the number of snapshots is 128 and, once again, 200 Monte Carlo runs are used to obtain each point.

Figure 5 shows the performance of conventional method and the proposed method; we can clearly see that the proposed method can distinguish the two signals while the conventional VAT fails for the $\mathrm{SNR}=10 \mathrm{~dB}$. The DOA finding results of proposed method is $\left(0.1^{\circ}, 5.2^{\circ}\right)$. The resolution and accuracy has been greatly improved compared conventional interpolated method. This is because the proposed method enhanced the signal subspace and improved the orthogonality between the signal and noise subspace by projection process. The proposed method is considerably more accurate than the conventional methods (also see Figure 4).

Figure 6 shows the root-mean-square errors (RMSEs) for the MUSIC-based DOA estimators versus SNR by using the conventional interpolation approach, the modified interpolation approach, and the real eight-antenna array. The CramerRao bounds (CRB) [18] of a real four-element array (CRB1) and eight-element array (CRB2) are plotted as a benchmark.

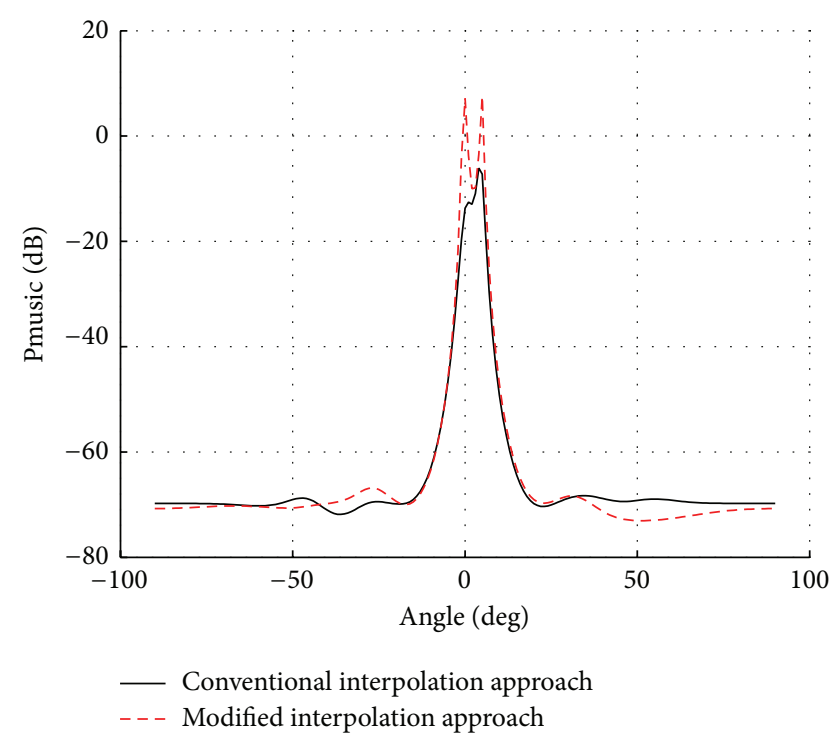

FIGURE 5: Spatial spectrum of MUSIC algorithm for two methods comparison.

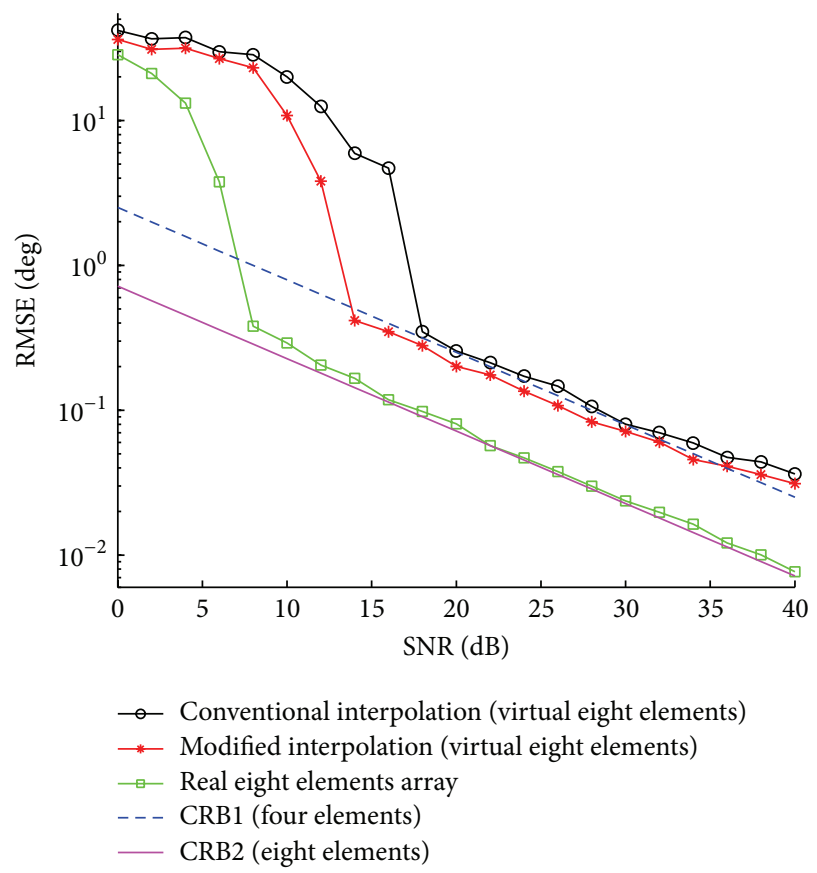

FIGURE 6: RMSE versus SNR for MUSIC-based DOA.

It can be observed from this figure that the proposed method has better RMSE performance than the conventional method for the entire range of SNR values. Since the interpolation transformation can increase the DOF of an antenna array, the DOF of the four-antenna array increases to seven in this example. When SNR $>15 \mathrm{~dB}$, the RMSE of modified interpolation is lower than CRB1 but is still larger than CRB2. The RMSE of the conventional interpolation approach is larger than CRB1.

The probabilities of source resolution versus SNR are shown in Figure 7 for different methods. It can be observed 


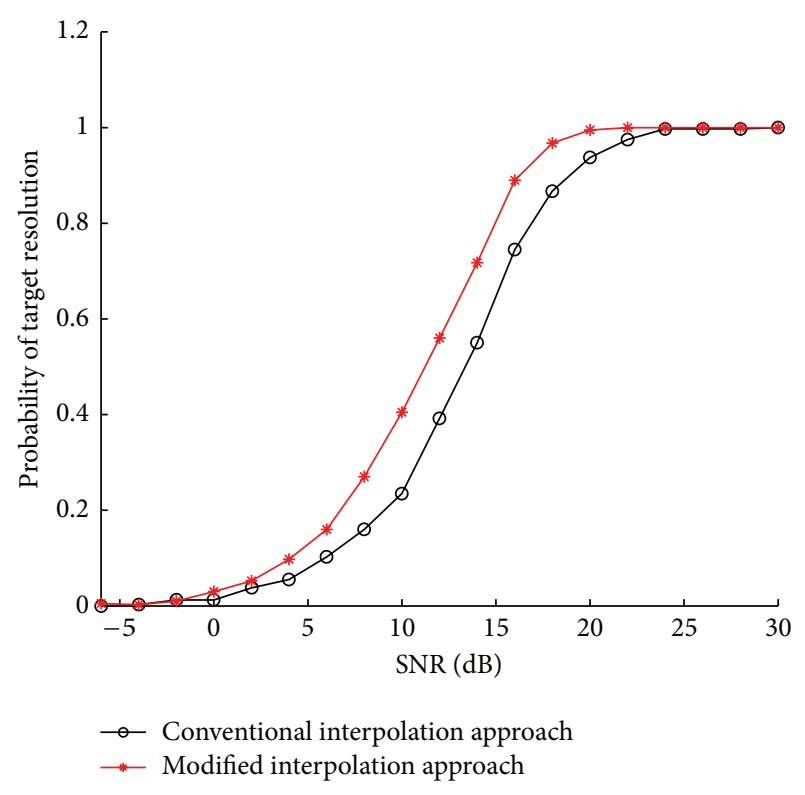

FIGURE 7: Probability of target resolution versus SNR.

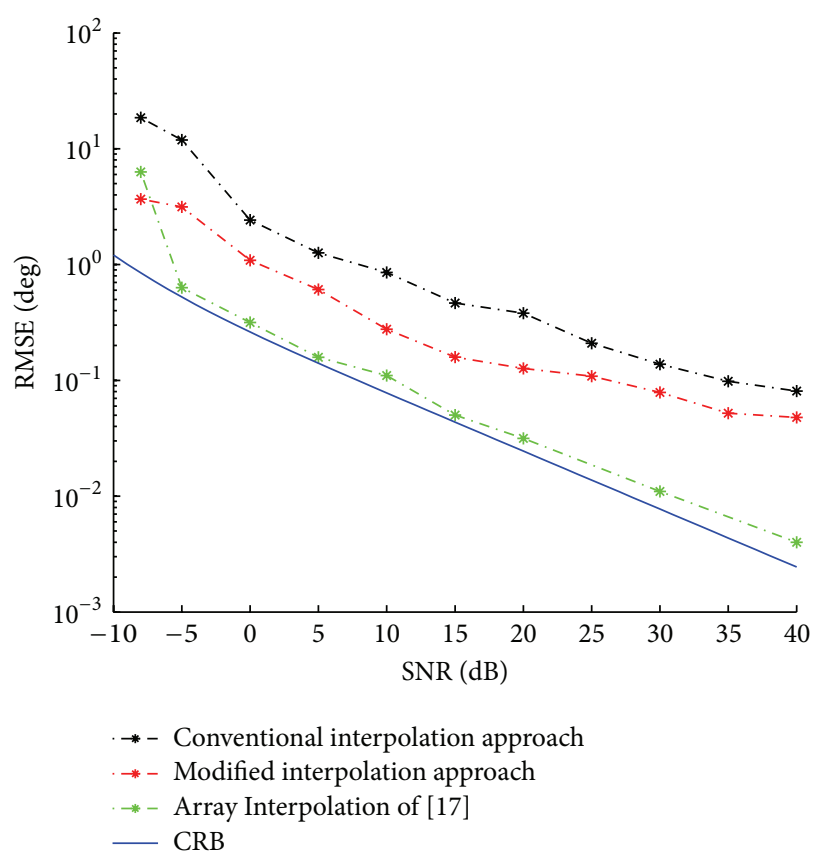

FIGURE 8: RMSE versus SNR.

results show that the proposed approach offers better estimation accuracy and has a high resolution performance compared to the tradition interpolation method.

\section{Conflict of Interests}

The authors declare that there is no conflict of interests regarding the publication of this paper.

\section{Acknowledgments}

This work was partially supported by "973" Basic Research Development Program of China (no. 6131380101). This paper is also supported by Pre-Research Fund of the 12th Five-Year Plan (no. 4010403020102) and Fundamental Research Funds for the Central Universities (HEUCFT1304).

\section{References}

[1] K. M. Reddy and V. Reddy, "Analysis of interpolated arrays with spatial smoothing," Signal Processing, vol. 54, no. 3, pp. 261-272, 1996.

[2] T. P. Bronez, "Sector interpolation of nonuniform arrays for efficient high resolution bearing estimation," in Proceedings of the IEEE International Conference on Acoustics, Speech and Signal Processing, vol. 5, pp. 2885-2888, April 1988.

[3] A. J. Weiss and M. Gavish, "Direction finding using ESPRIT with interpolated arrays," IEEE Transactions on Signal Processing, vol. 39, no. 6, pp. 1473-1478, 1991.

[4] A. J. Weiss, "Performance analysis of spatial smoothing with interpolated arrays," EEE Transactions on Signal Processing, vol. 41, no. 5, pp. 1881-1892, 1993. 
[5] D. N. Swingler and R. S. Walker, "Line-array beamforming using linear prediction for aperture interpolation and extrapolation," IEEE Transactions on Acoustics, Speech, and Signal Processing, vol. 37, no. 1, pp. 16-30, 1989.

[6] F. Belloni, A. Richter, and V. Koivunen, "DoA estimation via manifold separation for arbitrary array structures," IEEE Transactions on Signal Processing, vol. 55, no. 10, pp. 4800-4810, 2007.

[7] W. Li, Y. Li, and W. Yu, "On adaptive beamforming for coherent interference suppression via virtual antenna array," Progress in Electromagnetics Research, vol. 125, pp. 165-184, 2012.

[8] B. Friedlander, "Direction finding using an interpolated array," in Proceedings of the International Conference on Acoustics, Speech, and Signal Processing (ICASSP '90), vol. 5, pp. 2951-2954, Albuquerque, NM, USA, April 1990.

[9] B. Friedlander, "The root-MUSIC algorithm for direction finding with interpolated arrays," Signal Processing, vol. 30, no. 1, pp. 15-29, 1993.

[10] Y. S. Kim and Y. S. Kim, "Modified resolution capability via virtual expansion of array," Electronics Letters, vol. 35, no. 19, pp. 1596-1597, 1999.

[11] Y. Wang, H. Chen, and S. Wan, "An effective DOA method via virtual array transformation," Science in China Series E: Technological Sciences, vol. 44, no. 1, pp. 75-82, 2001.

[12] B. Friedlander and A. J. Weiss, "Direction finding using spatial smoothing with interpolated arrays," IEEE Transactions on Aerospace and Electronic Systems, vol. 28, no. 2, pp. 574-587, 1992.

[13] B. K. Lau, G. Cook, and Y. H. Leung, "A modified array interpolation approach to DOA estimation in correlated signal environments," in Proceedings of the IEEE International Conference on Acoustics, Speech, and Signal Processing, vol. 2, no. 2, pp. 237-240, 2004.

[14] P. Yang, F. Yang, and Z. P. Nie, "DOA estimation with sub-array divided technique and interporlated esprit algorithm on a cylindrical conformal array antenna," Progress in Electromagnetics Research, vol. 103, pp. 201-216, 2010.

[15] M. Pesavento, A. B. Gershman, and Z. Q. Luo, "Robust array interpolation using second-order cone programming," Signal Processing Letters, vol. 9, no. 1, pp. 8-11, 2002.

[16] P. Hyberg, M. Jansson, and B. Ottersten, "Array interpolation and bias reduction," IEEE Transactions on Signal Processing, vol. 52, no. 10, pp. 2711-2720, 2004.

[17] P. Hyberg, M. Jansson, and B. Ottersten, "Array interpolation and DOA MSE reduction," IEEE Transactions on Signal Processing, vol. 53, no. 12, pp. 4464-4471, 2005.

[18] P. Stoica and A. Nehorai, "MUSIC, maximum likelihood, and Cramer-Rao bound: further results and comparisons," IEEE Transactions on Acoustics, Speech and Signal Processing, vol. 38, no. 12, pp. 2140-2150, 1990. 

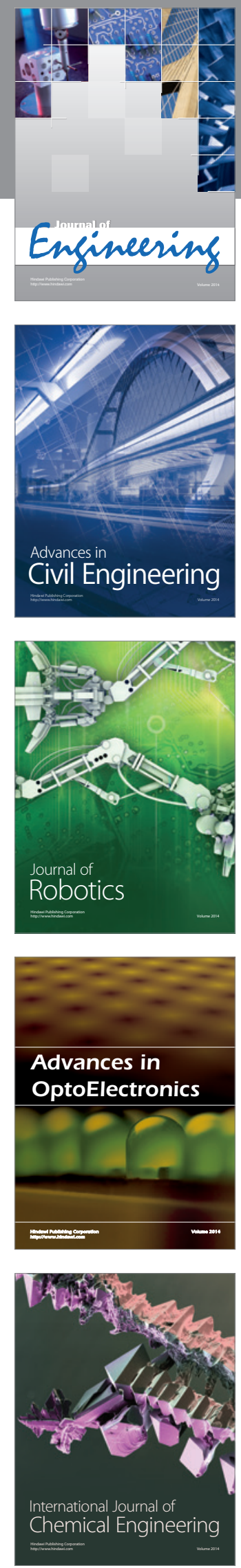

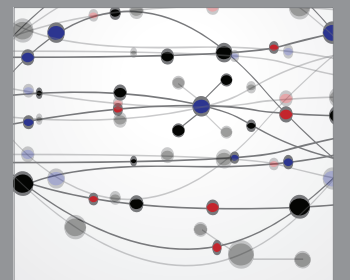

The Scientific World Journal
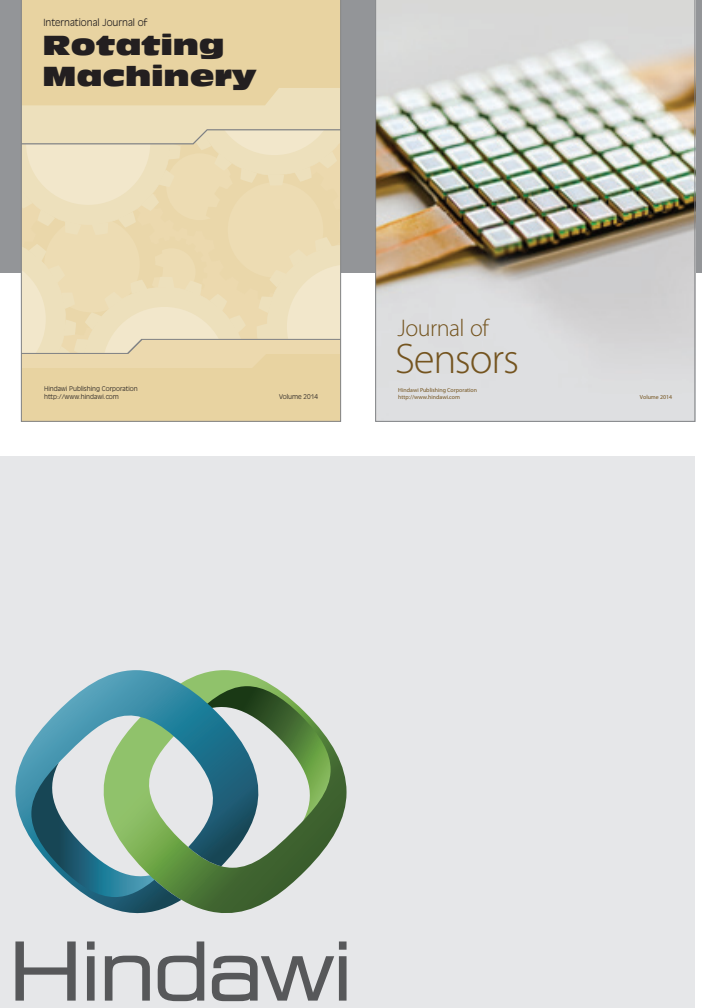

Submit your manuscripts at http://www.hindawi.com
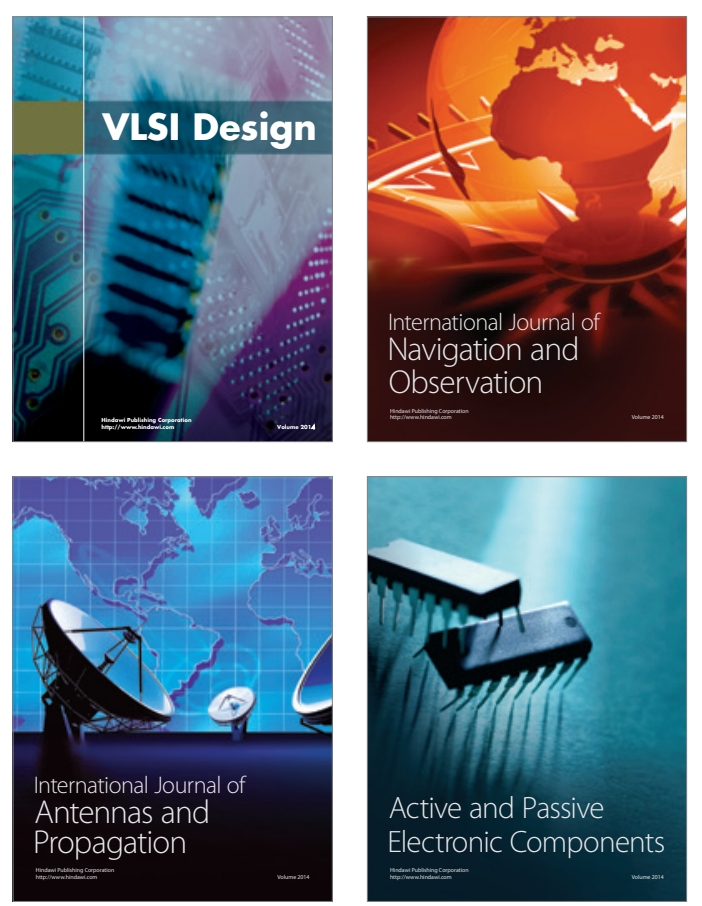
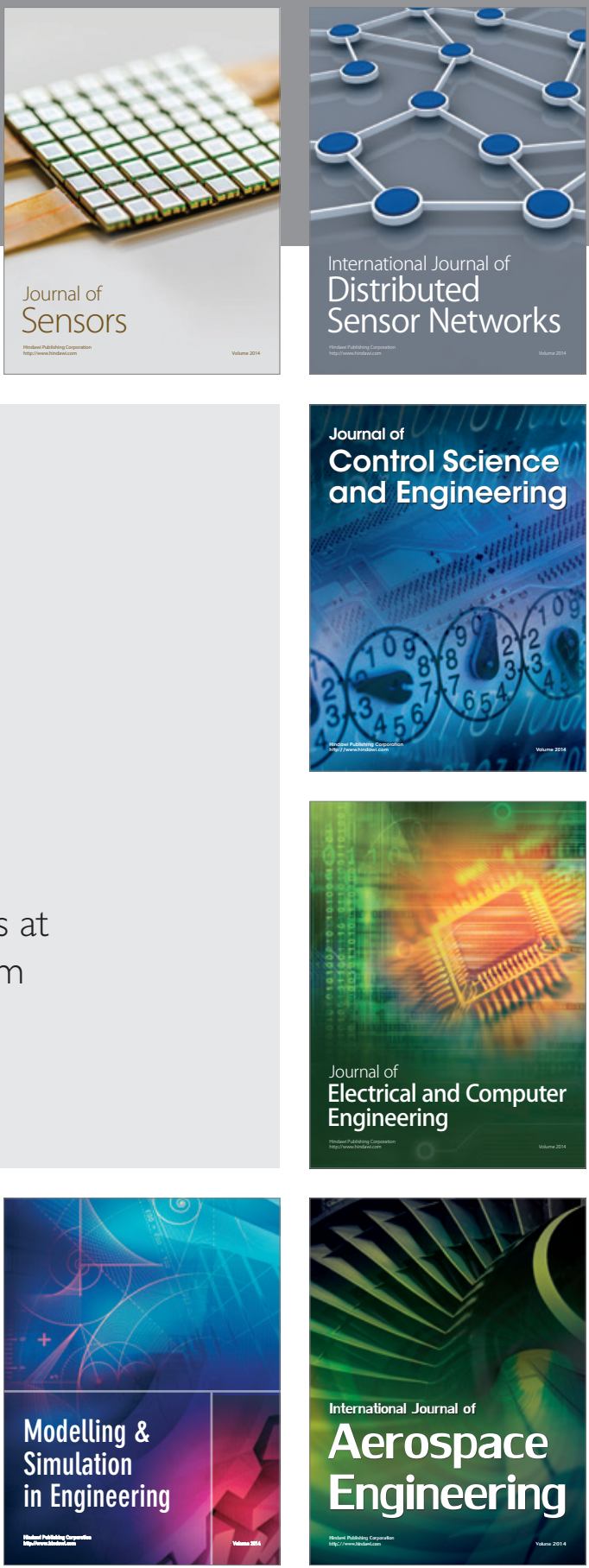

Journal of

Control Science

and Engineering
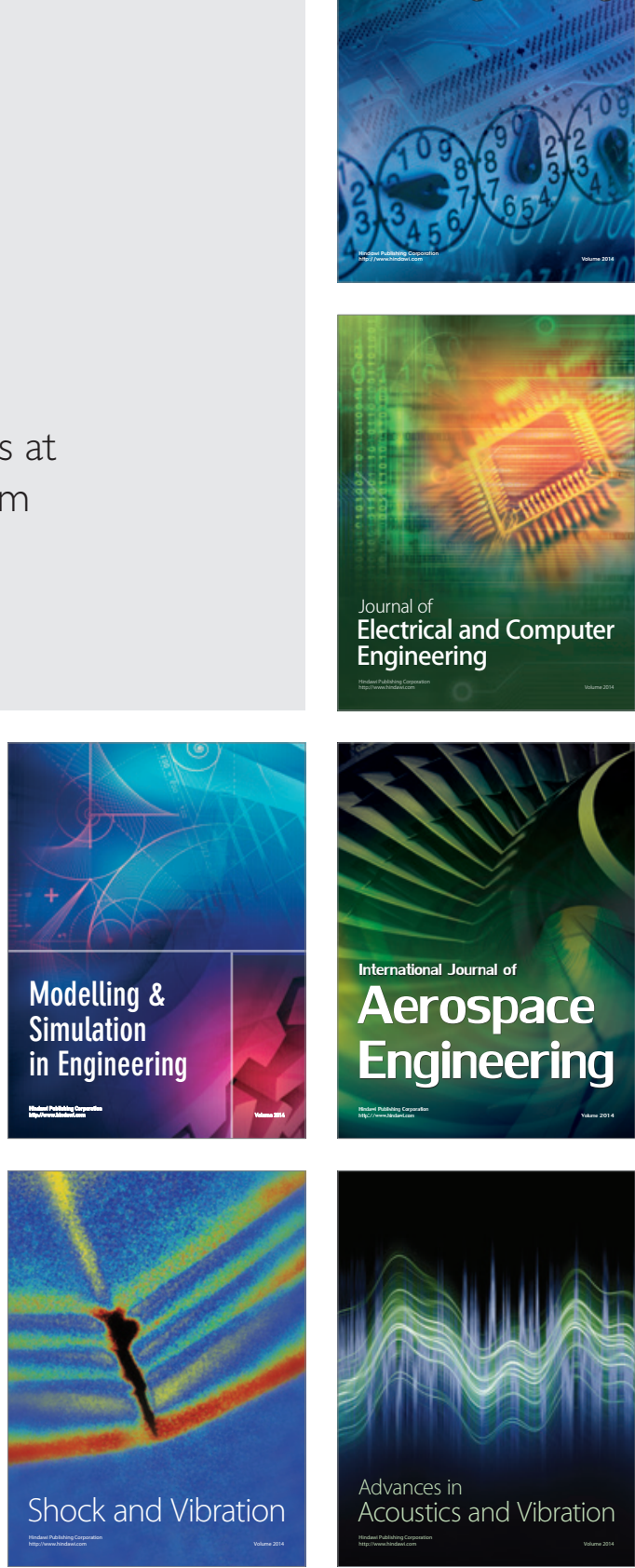